\title{
SUPERNOVAE FROM WOLF-RAYET STARS
}

\author{
NORBERT LANGER ${ }^{1}$ and STANFORD E. WOOSLEY ${ }^{2}$
}

1: Universitäts-Sternwarte Göttingen, F.R.G.

2: Lick Observatory, UC Santa Cruz, U.S.A.

The complete evolution of a star with an initial mass of $60 M_{\odot}$ and $\mathrm{Z}=\mathrm{Z}_{\odot}$ (i.e. a typical WR progenitor) from the ZAMS through the supernova phase has been investigated. Mass loss in the different evolutionary stages, especially mass dependent WR mass loss, leads to a WO star (surface mass fractions $\{\mathrm{He}, \mathrm{C}, \mathrm{O}\}=\{0.14$, $0.38,0.48\}$; cf. Fig. 1) of $4.2 M_{\odot}$ as pre-SN configuration. The low final mass may be typical for a wide range of initial masses (cf. Langer, 1989, Astr. Ap. 220, 135).

In the ensueing SN explosion $\left(1.6510^{51} \mathrm{erg}, v_{\max } \simeq 1.510^{9} \mathrm{~cm} \mathrm{~s}^{-1}\right) \sim 0.3 M_{\odot}$ radioactive ${ }^{56} \mathrm{Ni}$ are synthesized (Fig. 1). The reason for this large amount of nickel is a shallow density gradient in the pre-SN star, a consequence of its former large initial mass. Composition mixing was found to have only small effects on the peak width of the bolometric light curve. Effects of clumping, simulated by decreasing the $\mathrm{e}^{-}$-opacity, lead to an earlier and brighter maximum and a smaller peak width (Fig. 2).

We conclude that light curves of explosions of low mass WR stars, which originate from massive $\mathrm{O}$-stars, may resemble those of Type $\mathrm{Ib}$ supernovae. The large amount of radioactive ${ }^{56} \mathrm{Ni}$ and the spectroscopic signature of the mantel composition may discriminate our model from other proposed Type Ib progenitor scenarios.
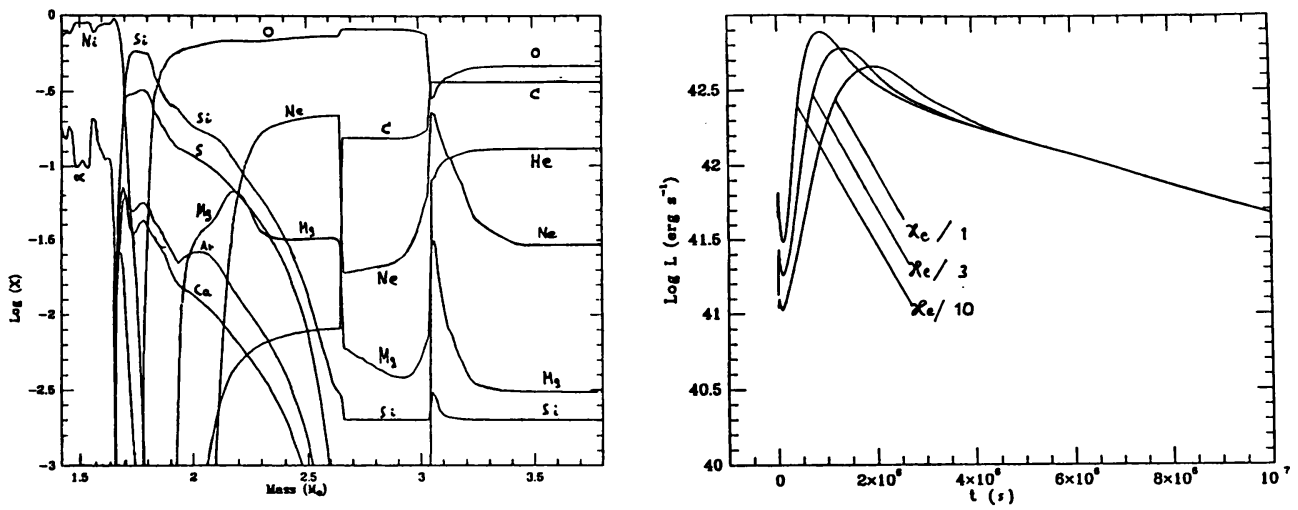

Fig.1 (left): Post-explosion composition of the considered 4.2 $M_{\odot}$ WR star prior to partial mixing (from: Woosley, Langer, Weaver, in prep.). Fig.2 (right): Bolometric light curve of the explosion of the partly mixed $4.2 M_{\odot}$ WR object for three different values of the $\mathrm{e}^{-}$-opacity (from: Woosley, Ensman, Langer, in prep.). 